\title{
Multicast Traffic Grooming in WDM Networks
}

\author{
Ahmed E. Kamal and Raza Ul-Mustafa \\ Department of Electrical and Computer Engineering, Iowa State University, Ames, IA, U.S.A.
}

\begin{abstract}
This paper considers the problem of grooming multicast traffic in WDM networks, with arbitrary mesh topologies. The problem is different from grooming of unicast traffic, since traffic can be delivered to destinations through other destinations in the same set, or through branching points. The paper presents an optimal Integer Linear Programming (ILP) formulation in order to minimize the cost of the network in terms of the number of SONET Add/Drop Multiplexers (ADM). The formulation also minimizes the number of wavelength channels used in the network, and does not allow bifurcation of traffic. Since the ILP formulation is able to solve limited size problems, the paper also introduces a heuristic approach to solve the problem.
\end{abstract}

Keywords: WDM networks; traffic grooming; ADMs; mesh topology; nonuniform traffic; multicast traffic; optimization; Integer Linear Programming (ILP); heuristics.

\section{INTRODUCTION}

During the last decade, Wavelength Division Multiplexing (WDM) Networks ${ }^{1}$ have emerged as an attractive architecture for backbone networks. WDM networks provide high bandwidth, on the order of tens of Gigabits per second per channel. However, recently two observations are driving the research community to explore the traffic grooming problem in WDM networks. First, the bandwidth requirements of most of the current applications are just a fraction of the bandwidth offered by a single wavelength in WDM networks. Second, the dominant cost factor in WDM networks is not the number of wavelengths but rather the network components, specifically, higher layer equipment, such as SONET Add/Drop multiplexers (ADMs), or MPLS or IP router ports *. Therefore the cost effectiveness of WDM networks depends on the amount of the optical passthrough provided by the network to the given traffic, thus reducing the number and cost of the higher layer equipment. However, the amount of the optical passthrough depends on the traffic arrangement on the optical layer. Traffic grooming is therefore defined as an intelligent allocation of the traffic demands, between different network nodes, onto an available set of wavelengths in such a way that reduces the overall cost of the network.

In general the traffic grooming problem is regarded to be even harder than the combined virtual topology design and Routing and Wavelength Assignment (RWA) problem. ${ }^{2}$ To make the problem somewhat less difficult, many relaxations have been considered in the literature. For example, most of the studies allow the traffic between each source-destination set to be (vertically) split over multiple wavelengths - a condition known as bifurcation. Due to bifurcation different components of the same traffic demand may traverse different links. This provision provides flexibility in traffic allocation, which may lead to a reduction in the number of wavelengths as well as the number of ADMs. Although allowing bifurcation simplifies the problem, it is unrealistic since it increases the complexity and the cost of traffic reassembly, and may also introduce jitter at the application layer. Many applications, especially real-time applications, require that their traffic be kept intact, i.e., without demultiplexing at the source, independent switching at intermediate nodes, and multiplexing at the destination. In this paper we have considered the case in which the traffic between each pair of nodes is not allowed to be bifurcated, i.e., traffic grooming without bifurcation. Please note that a non-bifurcated assignment does not necessarily mean that a specific traffic demand cannot traverse different wavelengths, rather in practice it may traverse many wavelengths before reaching the destination (a condition we refer to as horizontal splitting). However, the whole traffic demand needs to be intact on each wavelength it traverses.

Further author information: (Send correspondence to Ahmed E. Kamal)

Ahmed E. Kamal: E-mail: kamal@iastate.edu, Telephone: 15152943580

Raza Ul-Mustafa: E-mail: raza@iastate.edu, Telephone: 15152942295

${ }^{*}$ Without loss of generality, in this paper we will assume that the Synchronous Optical Networks (SONET) is being used as a higher layer in WDM networks. 
All studies of traffic grooming have exclusively dealt with unicast traffic. However, it is expected that a sizable portion of the traffic in future high performance networks will be multicast in nature, for example, multiparty conferencing, video distribution, network news distribution, collaborative processing, and web content distribution to proxies. ${ }^{3}$ Interestingly, most of the multicast service applications require only sub-wavelength capacity. For example, HDTV can work well with just $20 \mathrm{Mbps}$, while a normal TV channel typically requires less than $2 \mathrm{Mbps}$, when compressed using MPEG-2. Therefore, many such connections can be groomed together onto a single wavelength. Although the problem of all-optical multicasting, in which traffic demands require one or more wavelength channels each, has been treated in the literature, ${ }^{4}$ to the best of our knowledge, the problem of grooming of multicast traffic which has sub-wavelength demands has not been studied. In this paper we have addressed the problem of multicast traffic grooming in WDM networks. In Section 2, with the help of an example, we will show that using a Steiner Minimum Tree (SMT), as a minimum multicast tree, will not give an optimal solution in terms of ADMs. Therefore, while grooming multicast traffic, optimal multicast tree construction techniques used in Internet multicasting, or all optical multicasting, are not directly applicable.

Traffic grooming in WDM networks, recently, has started to receive attention. A few survey papers have been published in this area. ${ }^{5-7}$ The general problem of traffic grooming with arbitrary traffic has been proven to be NP-Complete in Ref. 8. Most of the work related to traffic grooming in WDM networks has an emphasis on reducing the number of required higher layer components. ${ }^{6}$ However, there are some notable exceptions in which factors like the network utilization are optimized..$^{9-11}$ Related work can be categorized based on the traffic patterns (uniform traffic, arbitrary/non-uniform traffic), network topology (unidirectional ring, bidirectional ring, random topology) and solution approaches (heuristics, ILP, bounds). Few authors considered specific traffic patterns on ring topology, for example, uniform traffic, all-to-all, all-to-one between all node pairs, ${ }^{8,12,13}$ while other researchers considered the arbitrary traffic between node pairs on a ring topology. ${ }^{2,14-17}$ Few studies considered only unidirectional rings, ${ }^{2,8}$ while other studies considered both unidirectional and bidirectional rings. ${ }^{12,14-17}$ Most of the researchers developed heuristic approaches to the problem, while many of them also focused on obtaining bounds on the number of ADMs required for specific topologies and traffic patterns. ${ }^{2,8,12-14}$ Optimal approaches are developed in Ref. 2 for unidirectional rings, and in Ref. 16 for bidirectional rings. Only a few studies considered arbitrary mesh topologies. ${ }^{11,18-20}$ It should be noted that ILP presented in Ref. 11 maximizes the network throughput on a mesh topology while ensuring non-bifurcation of traffic. However, that formulation guarantees non-bifurcation only in cases in which the capacity of a wavelength is an integer multiple of any of the traffic demands, which applies to all of the case studies presented in Ref. $11^{\dagger}$.

To the best of our knowledge, there is no work in the literature that considers the multicast traffic grooming problem. In this paper we will study the multicast traffic grooming problem with static traffic. This follows from the fact that optical networks are provisioned every few months, ${ }^{1}$ and as such the traffic can be considered static. We develop an ILP for the multicast traffic grooming problem, that can handle random topologies with arbitrary traffic (non-uniform multicast and unicast traffic), and also does not allow bifurcation. Our objective is to minimize the total cost of the higher layer equipment, which consists of the number of the ADMs, while using the least possible number of wavelengths. Moreover, we have developed a heuristic for solving large sized problems. The contributions of this paper to the traffic grooming problem in WDM networks can be summarized as follows.

- It introduces an ILP model for minimizing the network cost under multicast traffic grooming conditions.

- The formulation handles traffic grooming on random topologies without bifurcation of traffic demands.

- The introduced model optimally minimizes the cost of the WDM networks in terms of number of required ADMs, and simultaneously reduces the total number of required wavelengths (it is assumed that when a wavelength is used on at least one fiber link it is made available on all the fibers in the network). More will be said about the importance of this minimization later.

\footnotetext{
${ }^{\dagger}$ This can be shown by considering a counter example of a two node, one fiber network, where the fiber has two wavelengths. If the wavelength capacity is three units, and there are three demands between the two nodes, each of two units, all demands will be accommodated, hence allowing bifurcation.
} 


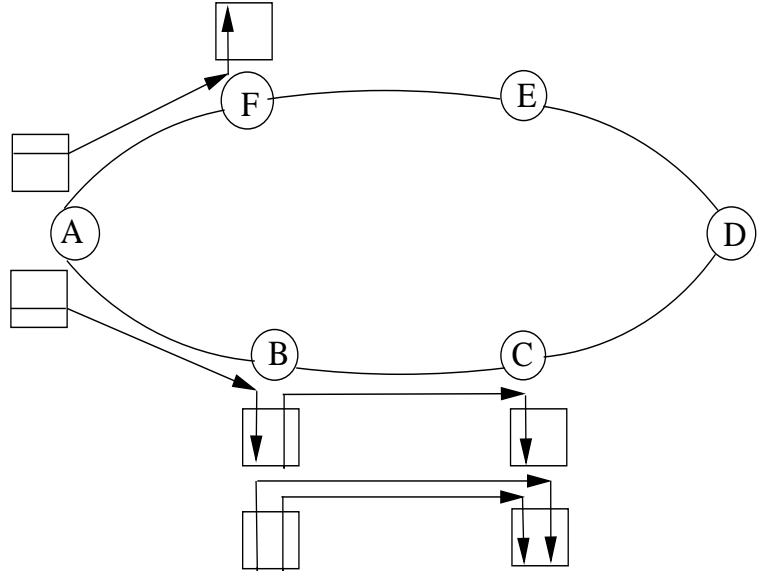

Figure 1. Routing using Steiner Minimum Tree.

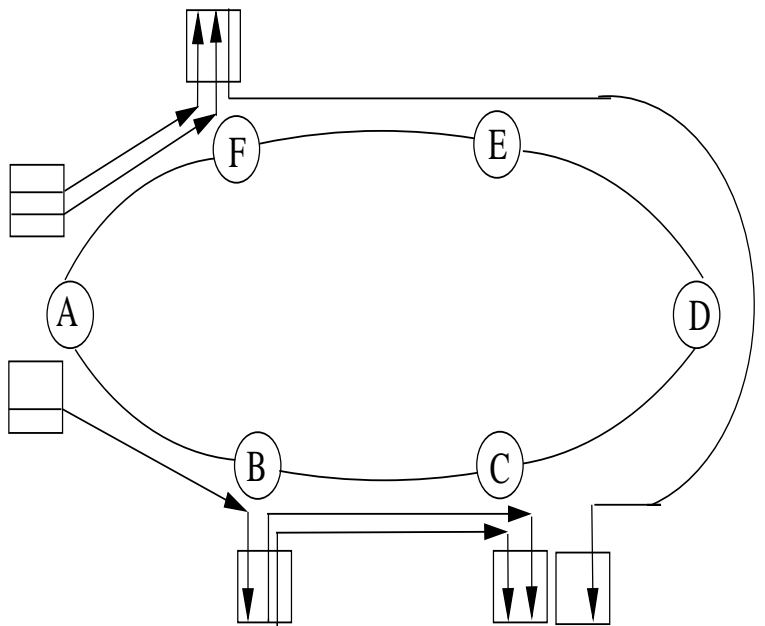

Figure 2. Routing in order to minimize the number of ADMs.

The rest of the paper is organized as follows. In Section 2, we present an ILP formulation for the multicast traffic grooming problem. Since the ILP method is only applicable to limited size networks, in Section 3, we present a heuristic approach to solving the problem, which can be applied to larger networks. In Section 4, we present the experimental results. Finally, Section 5 concludes the paper.

\section{PROBLEM FORMULATION}

In this section we will formulate an ILP for the multicast traffic grooming problem on random network topologies. We will start with the motivation for our model, followed by the model itself.

\subsection{Motivation}

In this section we will formulate an ILP for the multicast traffic grooming problem on random network topologies. As we are considering static traffic, the cost of the network can be drastically reduced by extensive off-line computation. Hence obtaining optimal solutions, even if it is compute-intensive, seems to be a logical choice, especially given that the addition/subtraction of few ADMs has a profound effect on the cost of network.

Before we present our ILP formulation, we will show, with the help of an example, that using a Steiner Minimum Tree (SMT) in terms of the number of hops, will not necessarily give an optimal solution in terms of the number of required ADMs on our problem. Consider a six-node bidirectional ring as shown in Figure 1. Let us assume that the capacity of each wavelength is 2 units and there exists 3 traffic sessions as follows.

Session 1: Source $=A$; Destination $=\{B, C\}$; Traffic demand $=1$ unit;

Session 2: Source $=B$; Destination $=\{C\}$; Traffic demand $=2$ unit;

Session 3: Source $=\mathrm{A}$; Destination $=\{\mathrm{F}\}$; Traffic demand $=1$ unit;

Routing the demands using an SMT requires 7 ADMs and two wavelengths, as shown in Figure 1. However, using the routing shown in Figure 2 costs just $6 \mathrm{ADMs}$ and one wavelength, which proves our claim.

One of the simple techniques applied in the literature to handle multicast traffic, is to treat every multicast demand as a set of unicast demands. However, such a policy will not lead to minimum number of ADMs in most cases. 


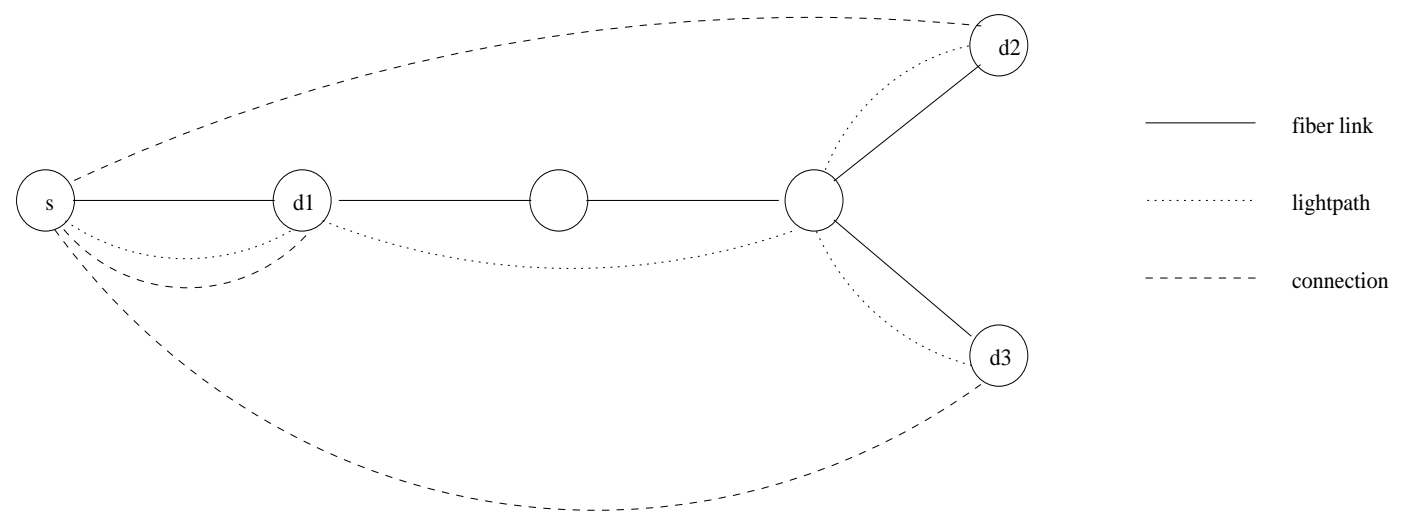

Figure 3. The three level network representation, source s, and destination set $d_{1}, d_{2}$ and $d_{3}$.

\subsection{The Model and its ILP}

In this subsection we will explain our model, define the terms used, and develop an ILP for it. We visualize the network at three different levels: physical level, lightpath level and connection level, as shown in Figure 3. The physical level represents the topology made of physical links between nodes, and is an input parameter. We assume that each physical link represents a bidirectional fiber that can support W wavelengths. The lightpath level represents the virtual topology made of all-optical lightpaths, and is an output from the ILP. Each lightpath can span several physical links. Please note that between any node pair more than one lightpaths may exist. Multiple such lighpaths may even exist on the same wavelength, because lightpaths can take different physical routes between the same node pair. Connection level links between nodes represent the traffic demand between such nodes. Each link at the connection level may span several lightpaths. With multicast traffic, we consider the case in which branching is implemented in the electronic domain, therefore, a multicast tree consists of several connection level links. For each member in a multicast group, the path over which delivery of traffic is made may involve other members of the same multicast. In Figure 3 nodes $d_{1}, d_{2}$ and $d_{3}$ are the members of the destination set of the multicast group originating at source node $s$.

Regarding notations, we will use $s$ and $d$ to represent source and destination of a connection, $i$ and $j$ to represent the source and destination nodes of a lightpath, $m$ and $n$ to represent the source and destination nodes of a physical link, respectively. In order to simplify notation, we let $K$ be the total number of sessions from all the sources. Then, each connection $c_{a}$, where $1 \leq a \leq K$, corresponds to an ordered pair $(s, k)$, where $k$ represents the $k^{t h}$ (unicast or multicast) session originating from source $s$. The destination set of session $c_{a}$ is $D_{c_{a}}$, and $d$ represents a destination in the destination set. The rest of the notations used in the paper are defined below.

$N$ : total number of nodes in the network

$W$ : number of wavelengths per fiber $\ddagger$

$g$ : Capacity of a wavelength in terms of number of basic units of traffic (also called grooming factor)

$\alpha$ : cost of an ADM

$\beta$ : cost of a wavelength

$Q$ : A very large integer number, (in our case $Q \geq N^{2}-N$ )

$F_{m n}$ : number of fibers connecting nodes $m$ and $n$

$$
F_{m n}= \begin{cases}F_{m n}=0, & \text { for non-adjacent nodes } \mathrm{m} \text { and } \mathrm{n} \\ F_{m n}=1, & \text { if and only if there is a direct physical fiber between nodes } \mathrm{m} \text { and } \mathrm{n}\end{cases}
$$

$F_{m n}^{i j, w}$ : number of lightpaths between node pair $(i, j)$ routed through fiber $(m, n)$ on wavelength $w$ $A D M_{n}$ : number of ADMs at node $n$

$L_{i j}$ : number of lightpaths from node $i$ to node $j$

\footnotetext{
${ }^{\ddagger}$ Although we start with $\mathrm{W}$ wavelengths, the ILP minimizes the total number of required wavelengths.
} 
$L_{i j}^{w}$ : number of lightpaths from node $i$ to node $j$ on wavelength $w$

$\psi$ : highest index of wavelengths used over all fiber links

$y_{w}$ : a binary indicator; must be 1 if there is at least one lightpath in the network on wavelength $w$.

$m_{c_{a}}$ : number of basic units of traffic required by connection $c_{a}$

$Z_{i j}^{c_{a}, d}$ : a binary indicator; is 1 if and only if connection $c_{a}$, destined to $d$, is employing a lightpath from $i$ to $j$ as an intermediate virtual link

$M_{i j}^{c_{a}}$ : a binary indicator; is 1 if and only if $\exists d \in D_{c_{a}}$, such that $Z_{i j}^{c_{a}, d}=1$. This means that connections $c_{a}$ is using lightpath $(i, j)$ to reach at least one destination.

$J_{i j}^{c_{a}, c_{b}}$ : a binary indicator; is 1 if and only if connection $c_{a}$ and $c_{b}$ are groomed on the same lightpath from $i$ to $j$.

Now we present the ILP for the multicast grooming problem. Our objective is to minimize the total cost of the higher layer equipment by minimizing the total number of the required ADMs, as well the total number of wavelengths used.

\section{Objective function:}

$$
\text { Minimize : } \alpha * \sum_{n} A D M_{n}+\beta * \psi
$$

In the objective function, $\alpha$ represents the cost of an $\mathrm{ADM}$, while $\beta$ represents the weight given to a wavelength, and is usually much smaller than $\alpha$.

\section{Subject to:}

- Number of ADMs:

The following two constraints ensure that for each originating or terminating lightpath an ADM is present at a node

$$
\begin{aligned}
& A D M_{i} \geq \sum_{w} \sum_{j, j \neq i} L_{i j}^{w} \quad \forall i \\
& A D M_{i} \geq \sum_{w} \sum_{j, j \neq i} L_{j i}^{w} \quad \forall i
\end{aligned}
$$

- Number of wavelengths:

The following two constraints ensure that $\psi$ will be the index of the highest numbered wavelength used in the network

$$
\begin{gathered}
\psi \geq w * y_{w} \quad \forall w \\
y_{w} \geq \sum_{i} \sum_{j, j \neq i} L_{i j}^{w} / Q \quad \forall w
\end{gathered}
$$

Notice that if wavelength $w$ is used in the network, then $y_{w}$ is 1 ; otherwise, $y_{w}$ may assume a value of 0 or 1 . However, if $w$ is not used and also wavelengths $X \geq w$ are not used, then $y_{w}=0$. Therefore, minimizing the $\psi$ will minimize the total number of different wavelengths in the network. The significance of this minimization is that it can result in using less expensive optical transceivers with wider spectral linewidths, e.g., MLM lasers instead of SLM lasers. Also, please note that minimizing the total number of the wavelengths in the network is different than minimizing the total number of the wavelengths between node pairs. The former is determined by the highest indexed wavelength between any node pair. Hence minimizing the total number of the wavelengths between node pairs still can have a sub-optimal solution in terms of the total number of the wavelengths used in the network.

- Lightpath level constraints:

$$
\sum_{w} L_{i j}^{w}=L_{i j} \quad \forall i, j
$$


The following constraint ensures that the origin node, $i$, of lightpaths $(i, j)$ has no incoming lightpaths between $i$ and $j$

$$
\sum_{m, f o r F_{m i}=1} F_{m i}^{i j, w}=0 \quad \forall i, j, w
$$

Similarly, the following constraint ensures that the destination node, $j$, of lightpaths $(i, j)$ has no outgoing lightpaths between $i$ and $j$

$$
\sum_{n, f \circ r F_{i n}=1} F_{j n}^{i j, w}=0 \quad \forall i, j, w
$$

The following constraint ensures wavelength continuity of lightpaths over multiple physical links by maintaining that for an intermediate node $x$ of lightpath $(i, j)$ on wavelength $w$, the number of incoming lightpaths is equal to the number of outgoing lightpaths

$$
\sum_{m, f \text { or } F_{m x}=1} F_{m x}^{i j, w}=\sum_{n, f \circ r F_{x n}=1} F_{x n}^{i j, w} \quad \forall w, i, j, x ; x \neq i, j
$$

Equations (10) and (11) state that the total number of lightpaths on wavelength $w$ to (from) node $j(i)$ of lightpath $(i, j)$ is equal to the total number of lightpaths between nodes $i$ and $j$ on wavelength $w$.

$$
\begin{aligned}
\sum_{m, f o r F_{m j}=1} F_{m j}^{i j, w} & =L_{i j}^{w} \quad \forall i, j, w \\
\sum_{n, \text { for } F_{i n}=1} F_{i n}^{i j, w} & =L_{i j}^{w} \quad \forall i, j, w
\end{aligned}
$$

Equations (9), (10) and (11) together ensure that for each lightpath there exists a corresponding physical path, while maintaining wavelength continuity over the physical path. Equation (12) below ensures that on a wavelength, $w$, on fiber $(m, n)$, only one lightpath is present.

$$
\sum_{i} \sum_{j, f o r F_{m n}=1} F_{m n}^{i j, w} \leq 1 \quad \forall m, n, w
$$

Since two or more multicast connections in the same destination set can share the same lightpaths, to reach their respective destinations, the bandwidth along the shared path could be shared too. The following three constraints ensure that the bandwidth of all lightpaths from $i$ to $j$ have not exceeded the physical capacity

$$
\begin{gathered}
M_{i j}^{c_{a}} \geq \sum_{d \in D_{c_{a}}} Z_{i j}^{c_{a}, d} / Q \quad \forall c_{a}, i, j \\
M_{i j}^{c_{a}} \leq \sum_{d \in D_{c_{a}}} Z_{i j}^{c_{a}, d} \quad \forall c_{a}, i, j \\
\sum_{c_{a}=1}^{K} m_{c_{a}} M_{i j}^{c_{a}} \leq L_{i j} * g \quad \forall i, j
\end{gathered}
$$

Note that for the bandwidth requirements of connection $c_{a}$ on lightpath $(i, j)$, using the $M_{i j}^{c_{a}}$ variables will avoid multiple counting of the same bandwidth $m_{c_{a}}$.

- Multicast connection topology constraints:

The following two constraints ensure that for the connection $c_{a}$ destined for $d$, no traffic is coming in (going out) the source (destination), respectively

$$
\sum_{i, i \neq s} Z_{i s}^{c_{a}, d}=0 \quad \forall c_{a}, d \in D_{c_{a}}
$$




$$
\sum_{j, j \neq d} Z_{d j}^{c_{a}, d}=0 \quad \forall c_{a}, d \in D_{c_{a}}
$$

The following two constraints ensure that the connection traffic between $s$ and $d$ is originating (terminating) at $s(d)$, respectively

$$
\begin{aligned}
\sum_{j, j \neq s} Z_{s j}^{c_{a}, d} & =1 \quad \forall c_{a}, d \in D_{c_{a}} \\
\sum_{i, i \neq d} Z_{i d}^{c_{a}, d} & =1 \quad \forall c_{a}, d \in D_{c_{a}}
\end{aligned}
$$

The following constraint preserves the continuity of connection traffic on multiple lightpaths

$$
\sum_{i, i \neq x} Z_{i x}^{c_{a}, d}=\sum_{j, j \neq x} Z_{x j}^{c_{a}, d} \quad \forall c_{a}, d \in D_{c_{a}}, x,(x \neq s, d)
$$

\section{- Non-Bifurcation:}

Note that equations (18), (19) and (20) together ensure that each traffic demand in the given traffic matrix is satisfied, using single or multiple lightpaths. However these equations alone do not ensure that bifurcation will not take place. Note that between any two nodes $i$ and $j$ there could exist more than one lightpath, even on the same wavelength, but using different physical routes. A demand can satisfy equations (18), (19) and (20) and still gets bifurcated over multiple lightpaths between nodes $i$ and $j$. This is because equation (20) constrains the traffic on all lightpaths between two nodes, without constraining the traffic on each lighpath.

To ensure that bifurcation does not take place, we need the following implication and an additional set of constraints

$$
J_{i j}^{c_{a}, c_{b}} \rightarrow M_{i j}^{c_{a}} \wedge M_{i j}^{c_{b}} \quad \forall c_{a}, c_{b}, i, j
$$

The implication in equation (21) means that if $J_{i j}^{c_{a}, c_{b}}$ is 1 (i.e., connections $c_{a}$ and $c_{b}$ share the same lightpath between node $i$ and node $j$ ), then both $M_{i j}^{c_{a}}$ and $M_{i j}^{c_{b}}$ must be 1 . The following equation captures the above implication

$$
J_{i j}^{c_{a}, c_{b}} \leq\left(M_{i j}^{c_{a}}+M_{i j}^{c_{b}}\right) / 2 \quad \forall c_{a}, c_{b}, i, j
$$

To ensure that the bandwidth of all the connections sharing the very same lightpath is within the capacity of that lightpath, we add the following constraint

$$
m_{c_{a}}+\sum_{c_{b}, c_{b} \neq c_{a}} m_{c_{b}} J_{i j}^{c_{a}, c_{b}} \leq g \quad \forall c_{a}, i, j
$$

Finally, the total number of shared and unshared lighpaths between nodes $(i, j)$ must be equal to the total number of lightpaths between these two nodes, as enforced by the following constraint

$$
L_{i j}=J_{i j}^{c_{1}, c_{1}}+\sum_{a=2}^{K}\left(J_{i j}^{c_{a}, c_{a}}-\vee_{b=1}^{a-1} J_{i j}^{c_{b}, c_{a}}\right) \quad \forall i, j
$$

The second term in equation (24) counts all the connections on a single lightpath only once, since if $\vee_{b=1}^{a-1} J_{i j}^{c_{b}, c_{a}}$ is 1 , then this means that connection $c_{a}$ is sharing a lightpath with a lower numbered connection, $c_{b}$. Therefore, subtracting this value from $J_{i, j}^{c_{a}, c_{a}}$ will avoid multiple counting. Minimizing the total number of ADMs in the objective function, would also minimize the $\sum_{i} \sum_{j} L_{i j}$. Equation (24) thus tries to maximize the sharing of each lightpath, while equation (23) will act as a check that sharing of each lightpath does not exceed its physical capacity.

For the sake of completeness we would like to mention that one can implement the disjunction operation, say, $C=\vee_{i=1}^{n} A_{i}, C \in\{0,1\}$, using the following two constraints; $C \leq \sum_{i=1}^{n} A_{i}$ and $C \geq \sum_{i=1}^{n} A_{i} / n$. These two constraints are used to obtain $\vee_{b=1}^{a-1} J_{i j}^{c_{b}, c_{a}}$ which is needed in equation (24). We would like to mention that our constraints involving the $F_{i n}^{i j, w}$ variables are similar to those in. ${ }^{11}$ 


\section{A HEURISTIC APPROACH}

In this section we will present our heuristic approach to solve the multicast traffic grooming problem. Although the ILP formulation yields an optimal solution, the computation time increases drastically with the increase in the problem size. Hence one has to resort to heuristic approaches for large sized problems. The quality of the solution obtained by the heuristic approach can be evaluated by comparing the results with the optimal results obtained from an ILP for small to medium sized problems.

Any solution method for the multicast traffic grooming problem must address the following sub-problems:

- routing of unicast traffic demands

- construction of trees for multicast traffic demands

- wavelength assignment of selected routes

- grooming of traffic demands onto wavelengths

The results of the ILP verify our claim that SMT will not lead to an optimal solution in terms of the number of the ADMs. We found many connections routed along non-shortest paths at the physical topology level. Our approach is to first obtain an initial solution using simple routing, wavelength assignment and grooming strategies and then improve that initial solution by exploring other possible routes and wavelength assignments for the multicast tree. The heuristic is shown in Figure 4.

The algorithm starts by constructing Shortest Path Tree (SPT) for each request $c_{a}$, which approximates an SMT. The SPT may consist of multiple hops, where each hop corresponds to a lightpath, which is routed over the shortest physical path. Each traffic request is then routed on the SPT using the first-fit (first-accommodating) wavelength. If no wavelength is available, a new wavelength is added. The corresponding number of required ADMs is computed. An iterative approach is then used to improve this initial solution. For each request, $c_{a}$, and for each of its destinations $d$, many alternative routes(paths) are explored and corresponding saving in terms of the number of the ADMs, had the request be routed on these alternative routes, is computed (lines 7-17). Among all the alternative routes, the route that gives the maximum saving (BestSaving) is selected as the best alternative route for that connection. If the computed maximum saving is above zero the traffic demand is re-routed on the best alternative path, and the path tree (which is no longer a shortest-path) of the request $c_{a}$ is updated. The complexity of the heuristic is $O\left(K N^{3} \log N\right)$, where $K$ is the total number of requests, and $N$ is the total number of the nodes.

\section{EXPERIMENTAL RESULTS}

In this section we will present the results of the ILP model and heuristic. The network used in the experiments consists of 6 nodes, and is shown in Figure 5. The traffic demands consist of integer multiples of OC-1 connections. The capacity of a wavelength, i.e., the grooming factor, is equivalent to OC- 48 .

The ILP problem is solved using Cplex. ${ }^{21}$ The values of $\alpha$ and $\beta$ are selected to be 100 and 1 , respectively. A sample traffic that consists of a mix of multicast and unicast sessions is generated and is shown in Table 1. The problem is run using six wavelengths. However, the optimal solution reduced the total number of wavelengths to three. The total number of the ADMs required is 22. The number of ADMs required at node $0,1,2,3,4$, and 5 are $4,2,5,3,3$, and 5 , respectively. The last column of the Table 1 shows the accommodating lightpaths for the corresponding multicast or unicast traffic demands. Notice that at the lighpath level, the multicast traffic is delivered either directly, from the source to the destination, or through another destination in the multicast destination set. Moreover, instead of establishing multiple individual lightpaths between a source and each of its destinations, many lightpaths carry the (multicast) traffic to more than one destination in the same session, while simultaneously grooming the traffic to other sessions. For example, for the multicast connection from source 0 to the destination set $\{1,2,4\}$, lightpath $(0-2)$ is carrying the traffic for all the destinations in the destination set, and simultaneously grooming the traffic from other sessions too. Although, the physical paths used by the lighpaths are not shown, an inspection of the results shows that not all the lighpaths are routed over 


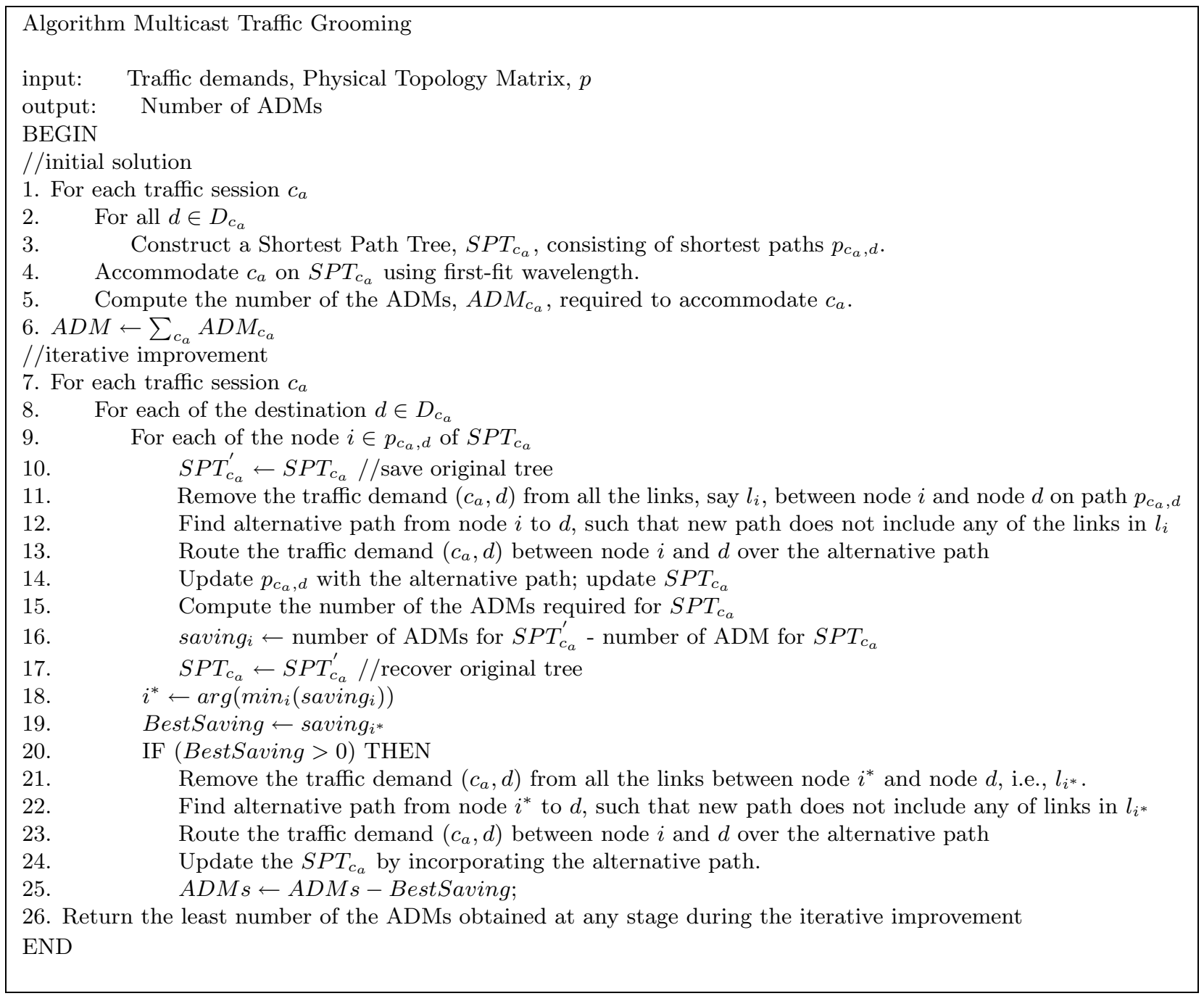

Figure 4. Multicast traffic grooming heuristic.

shortest physical path. For example, the shortest physical path for lighpath $(0-2)$ involves two physical hops, however, the optimal solution routes the lighpath $(0-2)$ over three physical hops, namely, $(0-3)$, $(3-1)$, and $(1-2)$. This shows that to obtain the minimum number of ADMs, one needs to explore routes other than the shortest-path routes. The number of the ADMs obtained by the heuristic for the same example is 30 . For other medium sized examples, the heuristic solution is within $25-30 \%$ of the optimal solution.

The NSF network, which consists of 14 nodes, is selected to run the heuristic for large sized problems. Table 2 shows the results of the experiments conducted on the NSF network topology and randomly generated traffic with the following parameters:

- Number of sessions from each node is generated uniformly between 0 and 14 .

- $50 \%$ of the sessions carry multicast traffic, while the remaining sessions carry unicast traffic.

- For each multicast session, the destination set size is uniformly distributed between 2 and 8 for the first scenario, and between 7 and 13 for the second scenario. 


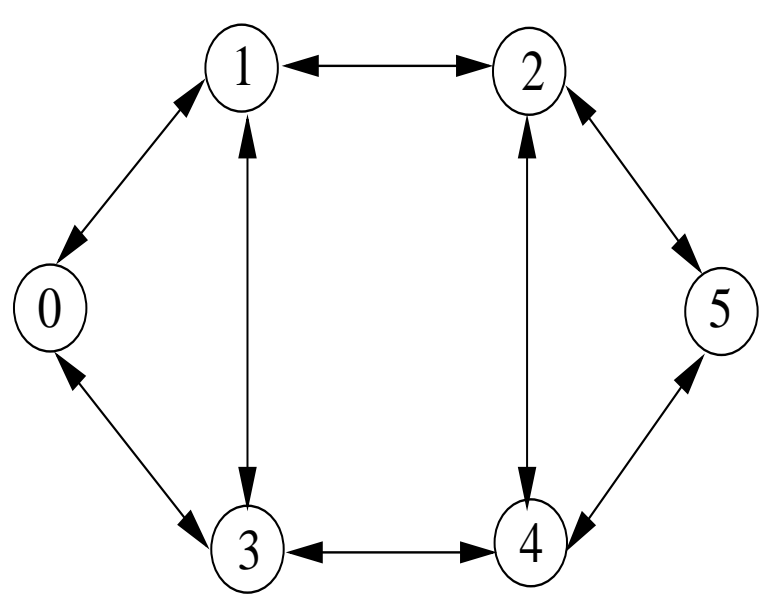

Figure 5. A six node network.

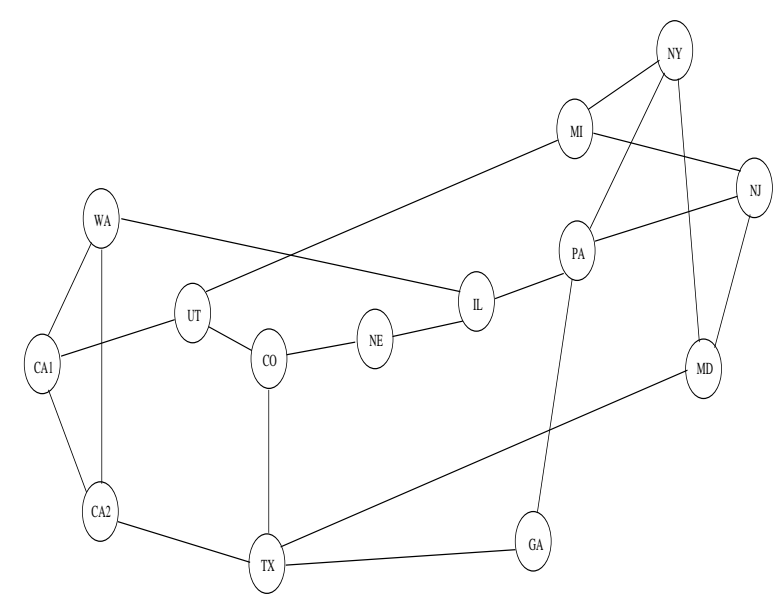

Figure 6. NSF Network topology.

Table 1. Traffic demands and the corresponding lightpaths obtained by the ILP.

\begin{tabular}{|ccc|c|}
\hline \hline Source & Destination set & Traffic (multiples of OC-1) & Lightpaths \\
\hline 0 & $\{1,2,4\}$ & 24 & $0-2,2-1,2-4$ \\
& $\{1,2,5\}$ & 6 & $0-2,2-1,1-5$ \\
& $\{2,3,4,5\}$ & 48 & $0-5,5-2,5-3,5-4$ \\
& $\{5\}$ & 12 & $0-5$ \\
\hline 1 & $\{0,2,5\}$ & 36 & $1-5,5-0,0-2$ \\
& $\{3,5\}$ & 18 & $1-3,3-5$ \\
& $\{3\}$ & 24 & $1-3$ \\
\hline 2 & $\{0,3,5\}$ & 24 & $2-3,3-0,3-5$ \\
& $\{1\}$ & 9 & $2-1$ \\
& $\{5\}$ & 18 & $2-5$ \\
& $\{0,1,3,4,5\}$ & 12 & $2-3,2-4,2-5,3-0,0-1$ \\
\hline 3 & $\{0,1\}$ & 3 & $3-0,0-2,2-1$ \\
& $\{4,5\}$ & 48 & $3-5,5-8$ \\
\hline 4 & $\{0\}$ & 24 & $4-0$ \\
& $\{0,1,2\}$ & 36 & $4-2,2-0,0-1$ \\
& $\{0\}$ & 24 & $4-0$ \\
& $\{2\}$ & 48 & $4-2$ \\
\hline \hline
\end{tabular}

- The destinations are chosen randomly among all the nodes, excluding the source, for both unicast and multicast sessions.

- The generated traffic, for both unicast and multicast sessions, is integer multiples of OC-1, and is uniformly chosen from the set $\{1,3,9,12,18,24,36,48\}$. These values represent the recommended rates for OC streams.

For comparison purposes, experiments are conducted for the original traffic load, generated as mentioned above, and also by doubling the load. Moreover, the results from the heuristic are compared with the case when all the multicast traffic is accommodated using multiple unicast connections. In Table 2 the two scenarios correspond to different destination set sizes. The total amount of the traffic generated for the first scenario is equivalent to 504 OC-1 streams, while the total amount of the traffic generated for the second scenario is equivalent to 924 OC-1 streams. From Table 2 it is evident that both the number of the ADMs and the number of the wavelengths increases by almost $100 \%$ when the load is doubled (the doubled load for the first and second scenario is equivalent to 1008 OC-1 streams and 1848 OC-1 streams, respectively). This shows that our 
Table 2. The number of the ADMs and wavelengths, obtained by the heuristic and by accommodating the traffic demands using multiple unicast connections.

\begin{tabular}{|c|c|l|l||l|l|}
\cline { 3 - 6 } \multicolumn{2}{c|}{} & Original load & Doubled load & $\begin{array}{l}\text { Multiple } \\
\text { unicasts with } \\
\text { original load }\end{array}$ & $\begin{array}{l}\text { Multiple } \\
\text { unicasts with } \\
\text { doubled load }\end{array}$ \\
\hline $\begin{array}{c}\text { First } \\
\text { Scenario }\end{array}$ & ADMs & 237 & 462 & 321 & 631 \\
\cline { 2 - 6 } & wavelengths & 15 & 30 & 34 & 68 \\
\hline \hline $\begin{array}{c}\text { Second } \\
\text { Scenario }\end{array}$ & ADMs & 381 & 741 & 630 & 1229 \\
\cline { 2 - 6 } & wavelengths & 19 & 37 & 59 & 117 \\
\hline
\end{tabular}

heuristic is grooming the traffic effectively, otherwise by doubling the load the increase in the required resources would have been less than twice. Moreover, Table 2 shows that when the multicast traffic is accommodated by employing multiple unicast connections, the number of the ADMs required is more than $30 \%$ than that of our heuristic. Similarly, using multiple unicast connections one ends up spending more than $100 \%$ wavelengths than that of our heuristic to accommodate the very same traffic demands. Finally the comparison between the first and the second scenario shows that if the traffic is accommodated by constructing the multicast trees and the size of the destination set is doubled, the number of the required ADMs increases by almost $60 \%$, while the increment in the number of wavelengths is less than twice. However, if multiple unicast connections are employed to accommodate the traffic, then doubling the size of the destination set increases the number of the ADMs by almost $100 \%$ while the number of the wavelengths increases by almost $75 \%$. Thus, our heuristic has a clear advantage over considering the multicast traffic as multiple unicast connections and then grooming them together.

Finally, to demonstrate that the proposed heuristic can run for even larger networks, we conducted experiments by randomly generating large networks of 30 and 50 nodes. The graph density, in terms of the number of the edges, is $O(N * \log N)$. The experiments are conducted with $\mathrm{g}=96$ and $\mathrm{g}=192$. Each node is a source for 20 sessions. Half of the sessions carry multicast traffic, while the remaining half of the sessions carry unicast traffic. For multicast traffic, the size of the destination set is uniformly distributed between 2 and 15 for 30 nodes experiment and between 2 and 25 for 50 nodes experiment. The generated traffic for both unicast and multicast sessions is integer multiples of OC-1, and is chosen from the set $\{1,3,9,12,18,24,36,48,96\}$ and $\{1,3,9,12,18,24,36,48,96,192\}$, for $\mathrm{g}=96$ and $\mathrm{g}=192$ experiments, respectively. The results are summarized in Table 3 .

Table 3. The number of the ADMs and wavelengths, obtained by the heuristic, for randomly generated large size networks.

\begin{tabular}{|l|c|l|l|}
\cline { 3 - 4 } \multicolumn{2}{c|}{} & $\mathrm{g}=96$ & $\mathrm{~g}=192$ \\
\hline \multirow{2}{*}{$\mathrm{N}=30$} & ADMs & 645 & 1825 \\
\cline { 2 - 4 } & wavelengths & 17 & 35 \\
\hline \hline \multirow{2}{*}{$\mathrm{N}=50$} & ADMs & 657 & 1602 \\
\cline { 2 - 4 } & wavelengths & 22 & 26 \\
\hline
\end{tabular}

\section{CONCLUSIONS}

In this paper, we have addressed the multicast grooming problem. We have developed an ILP to achieve optimal solutions in terms of minimum number of ADMs while reducing the number of wavelengths at the same time. The ILP formulation considers a random topology and solves the problem without bifurcation. Moreover, with the given formulation different objective functions can be optimized. Also we have presented a heuristic approach to solve the problem. The heuristic first obtains an initial solution using shortest path tree and first-fit wavelength, and then iteratively improves it by exploring other routes. The numerical results show that optimal solutions can be obtained for limited size problems, while the heuristic approach can be used for large sized problems. 


\section{ACKNOWLEDGMENTS}

This work is supported in part by grant ANI-0087746 from the National science Foundation.

\section{REFERENCES}

1. R. Ramaswami and K. Sivarajan, Optical Networks: A Practical Perspective, Morgan Kaufmann, San Francisco, CA,, 2001.

2. R. Dutta and G. N. Rouskas, "On optimal traffic grooming in wdm rings," IEEE Journal on Selected Areas in Communications 20, pp. 110-121, 2002.

3. G. Feng, C. Siew, and T.-S. Yum, "Architectural design and bandwidth demand analysis for multiparty videoconferenceing on sonet/atm rings," IEEE Journal on Selected Areas in Communications 20, pp. 1580$1588,2002$.

4. G. N. Rouskas, "Optical layer multicast: Rationale, building blocks and challenges," IEEE Network 17, pp. 60-65, 2003.

5. E. H. Modiano, "Traffic grooming in wdm networks," IEEE Communications Magazine 39, pp. 124-129, 2001.

6. R. Dutta and G. Rouskas, "Traffic grooming in wdm networks: Past and future," IEEE Network 16, pp. 46$56,2002$.

7. K. Zhu and B. Mukherjee, "A review of traffic grooming in wdm optical networks: architecture and challenges," Optical Networks Magazine 4, pp. 55-64, 2003.

8. A. L. Chiu and E. H. Modiano, "Traffic grooming algorithms for reducing electronic multiplexing costs in wdm ring networks," IEEE Journal of Lightwave technology 18, pp. 2-12, 2000.

9. S. Thiagarajan and A. K. Somani, "Traffic grooming for survivable wdm mesh networks," in Proceedings of OPTICOMM'01, 2001.

10. R. Srinivasan and A. K. Somani, "Dynamic routing in wdm grooming networks," Photonic Network Communications 5, pp. 123-135, 2003.

11. K. Zhu and B. Mukherjee, "Traffic grooming in a wdm mesh network," IEEE Journal on Selected Areas in Communications 20, pp. 122-133, 2002.

12. O. Gerstel, P. Lin, and G. Sasaki, "Combined wdm and sonet design," in Proceedings of IEEE INFOCOM'99, 2, pp. 734-743, 1999.

13. O. Gerstel, R. Ramaswami, and G. Sasaki, "Cost-effective traffic grooming in wdm rings," IEEE/ACM Transactions on Networking 8, pp. 618-630, 2000.

14. X. Y. Li, L. W. Liu, P. J. Wan, and O. Frieder, "Practical traffic grooming scheme for single-hub sonet/wdm rings," in Proceedings of IEEE 25th Annual Conference on Local Computer Networks (LCN), pp. 556-564, 2000 .

15. X. Zhang and C. Qiao, "An effective and comprehensive approach for traffic grooming and wavelength assignment in sonet/wdm rings," IEEE/ACM Transactions on Networking 8, pp. 608-617, 2000.

16. J. Wang, W. Cho, V. Vermuri, and B. Mukherjee, "Improved approaches for cost-effective traffic grooming in wdm ring networks: ilp formulations and single-hop and multihop connections," IEEE Journal of Lightwave Technology 19, pp. 1645-1653, 2001.

17. R. Ul-Mustafa and A. E. Kamal, "Wdm network design with non-uniform traffic grooming," in Proceedings of Optical Network Design and Modelling, 2, pp. 965-984, 2003.

18. G. Sasaki and T. Lin, "A minimal cost wdm network for incremental traffic," in Proceedings of SPIE Conference on All-Optical Networking, 3843, 1999.

19. V. Konda and T. Chow, "Algorithm for traffic grooming in optical networks to minimize the number of transceivers," in Proceedings of IEEE Workshop on High Performance Switching and Routing, pp. 218-221, 2001.

20. M. Brunato and R. Battiti, "A multistart randomized greedy algorithm for traffic grooming on mesh logical topologies," in Proceedings of Optical Network Design and Modelling, 242, 2002.

21. http://www.ilog.com/products/cplex/. 